\title{
Avaliação da Força de Preensão Palmar e Qualidade de Vida de Crianças com Câncer Submetidas à Quimioterapia com Vincristina
}

doi: https://doi.org/10.32635/2176-9745.RBC.2018v64n3.29

\author{
Evaluation of Handgrip Strength and Children's Quality of Life with Cancer Submitted to Chemotherapy with Vincristine \\ Evaluación de la Fuerza de Asimiento Palmar y Calidad de Vida de Niños con Cáncer Sometidos a la Quimioterapia \\ con Vincristina
}

Thayze Bairros da Costa ; Michelle Hagi Frantzeski²; Daniela Meirelles do Nascimento ${ }^{3}$; Lauro José Gregianin ${ }^{4}$

Resumo

Introduçáo: Com a quimioterapia, os pacientes podem apresentar diversas alteraçóes; entre elas, polineuropatia e mudança na qualidade de vida, tanto à criança como aos familiares. Objetivo: Avaliar a força de preensão palmar e a qualidade de vida de crianças e adolescentes com câncer submetidos à quimioterapia com vincristina. Método: Trata-se de um estudo, no qual os pacientes responderam aos questionários de anamnese e PedsQL $L^{T M}$ 3.0 Câncer Module, em três momentos diferentes do tratamento. Um dos seus responsáveis foi convidado a responder ao mesmo questionário. A força de preensão palmar foi aferida por meio de um dinamômetro, nos mesmos momentos. Resultados: A amostra foi composta por sete pacientes com mediana 7 (5-15 anos); com predomínio de meninas, residentes em Porto Alegre, RS, e diagnóstico prevalente de leucemia linfoide aguda, internados na primeira semana após o diagnóstico de câncer. A força de preensão palmar apresentou redução significativa para ambos os membros ( $p=0,018$, membro superior direito; $p=0,030$, membro superior esquerdo). Apesar de não apresentar resultado significativo, na maioria dos domínios do questionário de qualidade de vida, ocorreu declínio nas respostas, principalmente nas dos pais. Conclusáo: A quimioterapia com vincristina reduz a força muscular periférica em pacientes com câncer, nos 30 primeiros dias. Em relação à qualidade de vida, não foi apresentada diferença significativa. Porém, dentro dos domínios, pôde-se perceber algumas alteraçôes. Sendo assim, fica clara a importância do acompanhamento contínuo da fisioterapia junto a uma equipe preparada para esses pacientes e seus familiares.

Palavras-chave: Força da Mão; Qualidade de Vida; Neoplasias; Criança; Adolescente.

\begin{abstract}
Introduction: With chemotherapy, patients may have several changes. Among them: polyneuropathy and change in the quality of life, both the children and the family. Objective: To evaluate the handgrip's power and the life quality in these children and teens with cancer undergoing to chemotherapy with vincristine. Method: This is a study in which patients responded the questionaries of anamnsis and PedsQL $L^{T M} 3.0$ Cancer Module in three different moments of the treatment. One of your responsible was invited to answer separately the same questionarie. The handgrip's power was measured by dynamometer, in the same moments. Results: The sample was composed for seven patients with average 7 (5-15 years); The most of them were girls, residing in Porto Alegre-RS, with a prevalent diagnostic of Acute Lymphoid Leukemia and who were hospitalized in the first week after the diagnosis of cancer. The handgrip's strength show significant reduction for all of the limbs $(p=0,0018$, right upper limb; $p=0,0030$, left upper limb). Although of not show significant results, in most areas of the questionnaire of quality of life ocurred decline in the answers, mainly in the parents awnsers. Conclusion: The chemotherapy with vincristine, decrease in the peripheral muscle strength in the patients with cancer, in the first 30 days. In relationship of quality of life, there was no significant diference, however we could perceive some trends. Therefore, it is clear the importance of the continuous monitoring of physiotherapy with a team prepared for these patients and their families.

Key words: Hand Strength; Quality of Life; Neoplasms; Child; Adolescent.
\end{abstract}

Resumen

Introducción: Con la quimioterapia, los pacientes pueden presentar varias modificaciones. Entre ellas: polineuropatía y cambio en la calidad de vida, tanto del niño y de los familiares. Objetivo: Evaluar la fuerza de asimiento palmar y la calidad de vida de niños y adolescentes con cáncer sometidos a la quimioterapia con vincristina. Método: Se trata de un estudio, en el cual los pacientes respondieron a los cuestionarios de Anamnesis y PedsQLTM 3.0 Cáncer Module, en tres momentos diferentes del tratamiento. Uno de sus responsables fue invitado a responder el mismo cuestionario. La fuerza de asimiento palmar fue evaluada por medio de un dinamómetro, en los mismos momentos. Resultados: La muestra fue compuesta por siete pacientes con mediana 7 (5-15 ańos); teniendo más niñas, residentes en Porto AlegreRS, con diagnóstico prevalente de leucemia linfoide aguda y que estaban internadas en la primera semana después del diagnóstico de câncer. La fuerza de asimiento palmar presentó una reducción significativa para ambos miembros ( $\mathrm{p}=0,018$, miembro superior derecho, $\mathrm{p}=0,030$, miembro superior izquierdo). A pesar de no presentar resultados significativos, en la mayoría de los dominios del cuestionario de calidad de vida ocurrió declinación en las respuestas, principalmente en la respuesta de los padres. Conclusión: La quimioterapia con vincristina reduce la fuerza muscular periférica en pacientes con cáncer, en los 30 primeros días. En cuanto a la calidad de vida, no se presentó una diferencia significativa. Sin embargo, dentro de los dominios se pode percibir algunos cambios. Por lo tanto, queda claro la importancia del acompańamiento continuo de la fisioterapia junto a un equipo preparado para esos pacientes y sus familiares.

Palabras clave: Fuerza de la Mano; Calidad de Vida; Neoplasias; Niño; Adolescente.

\footnotetext{
${ }^{1}$ Complexo Hospitalar da Santa Casa de Misericórdia de Porto Alegre. Porto Alegre (RS), Brasil. Orcid iD: https://orcid.org/0000-0002-8159-831X

${ }^{2}$ Centro Universitário Metodista IPA. Porto Alegre (RS), Brasil. Orcid iD: https://orcid.org/0000-0002-6432-7788

${ }^{3}$ Centro Universitário Metodista IPA. Porto Alegre (RS), Brasil. Orcid iD: https://orcid.org/0000-0002-2894-8237

${ }^{4}$ Hospital de Clínicas de Porto Alegre. Porto Alegre (RS), Brasil. Orcid iD: https://orcid.org/0000-0003-0788-7858

Endereço para correspondência:Thayze Bairros da Costa. Rua Monsenhor Veras, 94 - Santana. Porto Alegre (RS), Brasil. CEP 90610-010. E-mail: thayze.b@hotmail.com.
} 


\section{INTRODUÇÃO}

O câncer infantil corresponde a um grupo de várias doenças que têm em comum a proliferação descontrolada de células anormais e que pode ocorrer em qualquer local do organismo. Os tumores mais frequentes na infância e na adolescência são as leucemias, os do sistema nervoso central e linfomas. Também acometem crianças e adolescentes o neuroblastoma, tumor de Wilms, retinoblastoma, tumor germinativo, osteossarcoma e sarcomas ${ }^{1}$.

No Brasil, o câncer já representa a primeira causa de morte ( $8 \%$ do total) por doença entre crianças e adolescentes de 1 a 19 anos. Segundo o Instituto Nacional de Câncer José Alencar Gomes da Silva (INCA) ${ }^{1}$, a estimativa para o ano de 2018 foi de 12.500 novos casos.

Os objetivos do tratamento do câncer são: a cura, o prolongamento da vida quando essa náo é possível e a paliaçáo dos sintomas. Quando o tratamento (quimioterapia, radioterapia ou cirurgia) não pode resultar em cura, ele deve levar a uma melhora do bem-estar e da qualidade de vida ${ }^{2}$.

A vincristina é um quimioterápico que está presente em quase todos os protocolos e a grande limitação da utilização dessa droga é a neurotoxicidade baseada na dose, o que ocasiona episódios de polineuropatias ${ }^{3}$. Os corticoides são essenciais no tratamento quimioterápico desses pacientes, e também geram importantes efeitos colaterais quando utilizados em altas doses, podendo ocasionar importantes complicaçóes musculoesqueléticas como fraqueza muscular, miopatias, osteoporose, fraturas e osteonecrose ${ }^{4}$. Os pacientes acabam apresentando menores níveis de aptidão física e densidade mineral óssea, quando comparados a crianças hígidas ${ }^{5,6}$.

Esse tratamento geralmente leva a um tempo de internação longo, além de várias intervençôes terapêuticas, o que torna necessário e de fundamental importância uma atenção especial para os pacientes e seus familiares. O direito de brincar e a evoluçáo física, afetiva, social e pessoal devem ser reservados, para que o atendimento da equipe seja cada vez melhor, visando à promoçáo de uma qualidade de vida satisfatória ${ }^{7}$.

Ainda permanece controverso o momento do tratamento quimioterápico em que as crianças e/ ou adolescentes começam a apresentar alteraçáo na força muscular e na qualidade de vida. A partir dessas informaçóes, percebe-se a necessidade de monitorizar esses pacientes durante o tratamento quimioterápico e instituir um programa de fisioterapia, a fim de minimizar essas complicaçôes. Sendo assim, objetivou-se avaliar a força de preensão palmar e a qualidade de vida de crianças e adolescentes com câncer, submetidos à quimioterapia com vincristina.

\section{MÉTODO}

Trata-se de um estudo com crianças e adolescentes acompanhados por 30 dias, durante o tratamento com quimioterapia, aprovado pelo Comitê de Ética em Pesquisa do Hospital de Clínicas de Porto Alegre (HCPA) (CAAE: 53521615.0.0000.5327) e pelo Comitê de Ética em Pesquisa do Centro Universitário Metodista - IPA (CAAE: 53521615.0.3001.5308), seguido pela Resolução CNS 466/12. Foi obtida autorização do MAPI Research Institute para aplicação do questionário Pediatric Quality of Life ${ }^{\mathrm{TM}}$ (PedsQL $\left.{ }^{\mathrm{TM}}\right)$ Cancer 3.0 Module.

A coleta dos dados foi realizada no período de julho a outubro de 2016, sendo a amostra não probabilística. Foram considerados elegíveis crianças e adolescentes de ambos os sexos, com faixa etária entre 5 e 17 anos, em acompanhamento no Serviço de Oncologia Pediátrica do HCPA, entre o primeiro e o sétimo dia de internação, com a confirmação do diagnóstico de câncer e em condiçóes de realizar os testes propostos (dinamometria e o questionário). Foram incluídos os pacientes que concordaram em participar do estudo, após a leitura do Termo de Assentimento Livre e Esclarecido (TALE), e que possuíam a autorização dos responsáveis e que iriam iniciar o tratamento com vincristina (todos os pacientes faziam parte do mesmo protocolo quimioterápico). Foram excluídos da amostra os indivíduos que desistiram, por algum motivo, de participar da pesquisa durante a avaliação.

No primeiro momento, considerados os critérios de inclusão, as crianças e adolescentes foram submetidos à avaliação com uma ficha de anamnese contendo dados de identificação, exames laboratoriais, medicamentos utilizados e variáveis antropométricas; resultados de exames complementares do prontuário e sinais vitais do momento - frequência cardíaca (FC), frequência respiratória (FR) e saturação de oxigênio $\left(\mathrm{SpO}_{2}\right)$.

A partir disso, foi realizado o teste de força de preensão palmar, por meio do dinamômetro do tipo Gripper de Contagem Eletrônica da marca Camry ${ }^{\oplus}$, modelo EH0117 , e com capacidade de $90 \mathrm{~kg}$. O posicionamento foi indicado para o paciente, segundo a Sociedade Americana de Terapeutas da Máo (ASHT) ${ }^{7}$. Foram utilizadas três tentativas para obter a preensão palmar máxima, com um intervalo de 60 segundos entre cada uma, pois, dessa forma, não iria ocorrer declínio entre a primeira e a última mensuraçáo. A medida mais alta foi escolhida e os pacientes deveriam manter a força durante cinco segundos.

A avaliação da qualidade de vida foi realizada por meio do questionário PedsQL $L^{\mathrm{TM}}$ Cancer 3.0 Module, aplicado para criança ou adolescente e para um dos pais, que responderam separadamente, porém, simultaneamente. 
Esse é um questionário dividido por 27 itens distribuídos em oito domínios: dor e ferimentos (2 itens), náusea (5 itens), ansiedade frente ao procedimento (3 itens), ansiedade frente ao tratamento (3 itens), preocupaçóes (3 itens), dificuldades cognitivas (5 itens), percepção da aparência física (3 itens), comunicação (3 itens). A escala é constituída por cinco opçóes de respostas: 0 = "nunca", 1 = "quase nunca", 2 = "algumas vezes", 3 = "muitas vezes" e 4 = "quase sempre" (esses itens são invertidos e transformados em: $100,75,50,25,0)$. Nas versóes adaptadas para as crianças de 5 a 7 anos, foram elaboradas apenas três opções de resposta: "nunca", "algumas vezes", "quase sempre" (100, 50, 0), e utilizada uma Escala de Faces. Na soma final, uma pontuação mais baixa traduz pior qualidade de vida.

A avaliação completa teve duração de aproximadamente 30 minutos. A coleta foi realizada no HCPA nos seguintes períodos: $0-7^{\circ} \mathrm{dia}$ (pré-quimioterapia) (T1), $15^{\circ} \mathrm{dia}$ (T2) e $30^{\circ}$ dia (pós-quimioterapia) (T3), sendo os dois últimos momentos realizados ambulatorialmente ou durante a internação (caso a criança/adolescente ainda estivesse internado).

As variáveis quantitativas foram descritas por mediana (mínimo e máximo). As variáveis qualitativas foram descritas por frequências absolutas e relativas. Para a comparação entre os três momentos, o teste de Friedman, complementado pelo de Wilcoxon, foi aplicado. As associaçóes entre as respostas dos pais e crianças no questionário de qualidade de vida foram avaliadas pelo coeficiente de correlaçáo de Spearman. O nível de significância adotado foi de $5 \%(\mathrm{p}<0,05)$ e as análises foram realizadas no programa SPSS versão 21.0.

\section{RESULTADOS}

Foram avaliadas a força de preensão palmar e a qualidade de vida em sete crianças e adolescentes com câncer. A idade da amostra, por meio de mediana (mínimo e máximo), foi de 7 (5-15) anos. Dos indivíduos que compunham a amostra, seis eram do sexo feminino
$(85,7 \%)$. O diagnóstico predominante foi leucemia linfoide aguda (LLA), com $71,4 \%$. As demais variáveis estão apresentadas na Tabela 1.

Tabela 1. Caracterização da amostra

\begin{tabular}{l|c}
\multicolumn{1}{c|}{ Variáveis } & $\mathbf{n = 7}$ \\
\hline Idade (anos) - md (min-máx) & $7(5-15)$ \\
\hline Sexo $-\mathrm{n}(\%)$ & \\
\hline $\mathrm{M}$ & $1(14,3)$ \\
\hline $\mathrm{F}$ & $6(85,7)$ \\
\hline Diagnóstico - $\mathrm{n}(\%)$ & \\
\hline Leucemia linfoide aguda & $5(71,4)$ \\
\hline Outros & $2(28,6)$ \\
\hline Tempo do diagnóstico - $\mathrm{n}(\%)$ & \\
\hline 0 a 7 dias & $3(42,9)$ \\
\hline 8 a 30 dias & $3(42,9)$ \\
\hline$\quad$ Mais de 30 dias & $1(14,3)$ \\
\hline Procedência - $\mathrm{n}(\%)$ & \\
\hline$\quad$ Capital local & $6(85,7)$ \\
\hline Interior do Estado & $1(14,3)$ \\
\hline
\end{tabular}

Legendas: md (mediana); min (mínimo); máx (máximo).

Em relação à força de preensão palmar, destaca-se uma redução significativa para ambos os membros $(p=0,018$, membro superior direito; $\mathrm{p}=0,030$, membro superior esquerdo), (Tabela 2).

$\mathrm{O}$ PedsQL $L^{T M}$ Cancer 3.0 Module não apontou resultados significativos, talvez por conta do pequeno número de amostra coletado. Apesar disso, conseguiu-se verificar que ocorreu uma menor pontuação nas respostas da avaliação dos sete primeiros dias, para a avaliação dos 30 dias. Na maioria dos domínios do questionário, ocorreu diminuição gradativa em relação ao tempo das avaliaçôes, principalmente nas respostas dos pais. Já o autorrelato demonstra uma queda até o $15^{\circ}$ dia, apresentando uma melhora no $30^{\circ}$ dia. (Tabela 3 )

$\mathrm{Na}$ análise das associaçóes, encontrou-se resultado significativo entre as respostas dos pais e crianças no T2 ( $\mathrm{p}=0,04 ; \mathrm{r}=0,77)$ para o domínio "Ansiedade frente ao tratamento". Sendo assim, a resposta para a mesma

Tabela 2. Comparação dos dados antropométricos e da força de preensão palmar ao longo do tempo

\begin{tabular}{|c|c|c|c|c|}
\hline \multirow[t]{2}{*}{ Variáveis } & $\begin{array}{c}\text { 0-7 dias } \\
\text { T1 }\end{array}$ & $\begin{array}{c}15 \text { dias } \\
\text { T2 }\end{array}$ & $\begin{array}{c}30 \text { dias } \\
\text { T3 }\end{array}$ & \multirow[t]{2}{*}{$p$} \\
\hline & md (min-máx) & md (min-máx) & md (min-máx) & \\
\hline Peso (kg) & $29(18-62)$ & $28,3(18-57)$ & $28(17-59,8)$ & 0,56 \\
\hline $\mathrm{IMC}\left(\mathrm{kg} / \mathrm{m}^{2}\right)$ & $17,4(13,8-23,6)$ & $17,0(13,9-24)$ & $17,2(14,1-22)$ & 0,56 \\
\hline FPP Direita (kg) & $10,8(5,8-36,3)$ & $10(4,8-34,4)$ & $9,8(4-20,8) ¥$ & $0,01^{*}$ \\
\hline FPP Esquerda (kg) & $8,1(5,5-35,2)$ & $7,4(4,5-30,6) €$ & $7,8(4,3-20,9) ¥$ & $0,03^{*}$ \\
\hline
\end{tabular}

Legendas: md (mediana); min (mínimo); máx (máximo); índice de massa corporal (IMC); força de preensão palmar (FPP). *p $<0.05$ teste de Friedman; $¥$ p $<0.05$ T3-T1 teste de Wilcoxon; $€ \mathrm{p}<0,05$ T3-T2 teste Wilcoxon. 
Tabela 3. Comparação dos resultados de qualidade de vida nos três momentos de avaliação

\begin{tabular}{|c|c|c|c|c|}
\hline \multirow[t]{2}{*}{ Variáveis } & $\begin{array}{c}\text { 0-7 dias } \\
\text { T1 }\end{array}$ & $\begin{array}{c}15 \text { dias } \\
\text { T2 }\end{array}$ & $\begin{array}{c}30 \text { dias } \\
\text { T3 }\end{array}$ & \multirow[t]{2}{*}{$\boldsymbol{p}$} \\
\hline & md (min-máx) & md (min-máx) & md (min-máx) & \\
\hline \multicolumn{5}{|l|}{ Crianças } \\
\hline Qualidade de vida total & $1.775(950-2300)$ & $1.500(1.150-2.325)$ & $1800(1.150-2.475)$ & 0,36 \\
\hline Dores/Machucados & $150(50-200)$ & $200(150-200)$ & $150(100-200)$ & 0,27 \\
\hline Náuseas & $450(400-500)$ & $450(250-500)$ & $350(200-500)$ & 0,16 \\
\hline Ansiedade frente ao procedimento & $150(0-300)$ & $225(0-300)$ & $250(0-300)$ & 0,49 \\
\hline Ansiedade frente ao tratamento & $250(100-300)$ & $275(175-300)$ & $300(225-300)$ & 0,18 \\
\hline Preocupações & $150(50-250)$ & $100(0-175)$ & $150(50-300)$ & 0,43 \\
\hline Dificuldades cognitivas & $350(150-500)$ & $325(100-450)$ & $325(200-500)$ & 0,86 \\
\hline Percepção da aparência física & $225(100-300)$ & $200(50-270)$ & $150(100-300)$ & 0,46 \\
\hline Comunicação & $200(0-275)$ & $150(0-275)$ & $125(50-275)$ & 0,73 \\
\hline \multicolumn{5}{|l|}{ Pais } \\
\hline Qualidade de vida total & $1.625(1.075-2150)$ & $1.550(1.250-2.100)$ & $1.425(900-2.100)$ & 0,63 \\
\hline Dores/Machucados & $150(100-200)$ & $175(0-200)$ & $175(100-200)$ & 0,95 \\
\hline Náuseas & $500(250-500)$ & $350(200-500) \pi$ & $300(0-400) ¥$ & $0,01^{*}$ \\
\hline Ansiedade frente ao procedimento & $75(0-150)$ & $175(0-250)$ & $150(25-250)$ & 0,22 \\
\hline Ansiedade frente ao tratamento & $100(0-300)$ & $150(125-300)$ & $225(150-300)$ & 0,09 \\
\hline Preocupações & $150(0-300)$ & $150(25-250)$ & $100(25-300)$ & 0,95 \\
\hline Dificuldades cognitivas & $325(125-500)$ & $350(275-400)$ & $350(225-500)$ & 0,85 \\
\hline Percepção da aparência física & $175(0-250)$ & $150(125-300)$ & $200(25-250)$ & 0,55 \\
\hline Comunicação & $125(50-250)$ & 150 (75-250) & $125(25-300)$ & 0,31 \\
\hline
\end{tabular}

Legendas: Mediana (md); Mínimo (min); Máximo (máx); ${ }^{*} \mathrm{p}<0.05$ teste de Friedman; $¥ \mathrm{p}<0.05$ T3-T1 teste de Wilcoxon; $\pi$ p $<0,05$ T2-T1 teste de Wilcoxon.

pergunta era dita de forma diferente entre as crianças e seus pais, na maioria dos domínios. Identificou-se que os pais percebem seus filhos com maiores dificuldades que a própria criança, porém esses dados não foram significativos assim como nos outros domínios.

\section{DISCUSSÃO}

O diagnóstico de maior prevalência nos pacientes avaliados foi de LLA, corroborando os dados do INCA ${ }^{1}$. Já em relação ao sexo predominante, vai contra alguns estudos $^{8,9}$ que apontam o gênero masculino como o mais prevalente.

No presente estudo, foi constatada a diminuição da força muscular por meio do teste de força de preensão palmar, a partir do momento em que iniciaram o tratamento quimioterápico até 30 dias após. Torna-se fundamental, segundo Moura ${ }^{10}$, a mensuração da força de preensão palmar, a fim de obter náo só o valor da integridade dos membros superiores, como dar uma ideia de força global, principalmente em indivíduos com doenças crônicas. Afinal, a partir disso, conseguiu-se interpretar os resultados estabelecendo objetivos adequados para o tratamento, visando à reabilitação principalmente nas atividades diárias desses pacientes.
O resultado do presente estudo se iguala ao de Götte ${ }^{11}$, que avaliou 47 pacientes de 6-17 anos durante o tratamento quimioterapêutico, no final da fase de indução. Foi realizado o Teste de Moon (para o desempenho motor na oncologia pediátrica), no qual uma das avaliaçóes foi a dinamometria para força de preensão palmar. Os resultados demonstraram uma redução significativa da força muscular durante a quimioterapia, em razão da inatividade e do tratamento, gerando déficits na performance motora, trazendo possivelmente problemas físicos e sociais para o futuro.

Os antineoplásicos estáo presentes no tratamento do câncer e, entre os protocolos farmacológicos utilizados nesse estudo, a vincristina estava incluída. É considerada a mais neurotóxica dos alcaloides da vinca, podendo causar fraqueza motora significativa das mãos e dos pés, em pacientes com efeitos tóxicos graves. Esses alcaloides constituem a base do tratamento das neoplasias malignas hematológicas há quase 50 anos $^{12}$.

Indo ao encontro dessas informaçóes, o estudo de Rodríguez-Reyes ${ }^{13}$ mostra que a neuropatia periférica induzida por vincristina está relacionada com a administração contínua desse medicamento, trazendo alguns sintomas como hipoestesia, fraqueza muscular de predomínio distal (pés e mãos), diminuição dos reflexos tendinosos e mialgias; ou seja, todas essas alterações 
acabam afetando diretamente a mobilidade desses pacientes, comprometendo assim a qualidade de vida.

No estudo de Díaz-Jaime ${ }^{14}$, pacientes com LLA, que utilizaram vincristina durante a quimioterapia, foram submetidos a um programa de fisioterapia, com avaliação do estudo de velocidades de neurocondução (VNC). Os 24 pacientes foram divididos em dois grupos: supervisionado (fisioterapia regularmente e acompanhado durante três meses) e sem supervisão (recebeu o programa de fisioterapia). Ambos realizaram VNC antes dos exercícios. Nos resultados, em $98 \%$ dos casos, o nervo mais afetado foi o fibular e, em $96 \%$, apresentou degeneração axonal desse mesmo nervo, sendo a neuropatia mais frequente. Sendo assim, o estudo evidencia a importância de um programa de exercícios vinculados à fisioterapia com supervisão, sendo realizados regularmente para minimizar os efeitos da quimioterapia nas neuropatias desenvolvidas durante ao tratamento.

No estudo de Hartman ${ }^{15}$, foi avaliada a força de alguns grupos musculares e o desempenho motor de 92 crianças tratadas com quimioterapia com vincristina, apresentando idade média de 8,9 anos e tempo médio desde o término do tratamento com 3,3 anos. Os autores verificaram diminuição significativa da força muscular dos dorsiflexores do tornozelo bilateralmente, flexores de punho do membro não dominante e da força de preensão palmar bilateralmente. $\mathrm{Na}$ avaliaçáo do desempenho motor, verificou-se que a diminuição da força muscular dos flexores de punho resultou em prejuízo da função da máo. $\mathrm{O}$ mesmo ocorreu com a força de preensão palmar que, em virtude da sua diminuição, provocou prejuízo do desempenho motor especificamente no aspecto habilidade com bola, não comprometendo os demais aspectos, como função da mão e equilíbrio.

Outro estudo foi realizado com o objetivo de avaliar os níveis de aptidão física e possíveis déficits no desempenho físico em 18 crianças com diagnóstico de LLA e linfoma, após dez meses de quimioterapia, comparadas a 40 crianças saudáveis. Foram realizados quatro testes; entre eles, a dinamometria. Ao final, Bianco ${ }^{6}$ concluiu que crianças com malignidades prévias apresentam um nível mais baixo de aptidão física, porém, mesmo assim, estâo aptas a participarem de intervençôes regulares de exercícios.

Deisenroth ${ }^{16}$ realizaram um estudo com o objetivo de avaliar o desempenho da força muscular e a qualidade de vida em crianças e adolescentes com câncer no início do tratamento primário. Seus achados mostram que há uma diminuição na qualidade de vida e força muscular desde o início do tratamento dessas crianças. Sugerindo acompanhamento fisioterapêutico para diminuição das morbidades.
A fim de minimizar o desconforto, os pacientes acabam diminuindo o nível de atividade física. Sendo assim, associando o tratamento ao repouso prolongado, acabam apresentando redução da massa muscular, influenciando na função muscular, pulmonar, incapacidade, perda de força, afetando diretamente a qualidade de vida ${ }^{17}$.

A quimioterapia acaba trazendo junto a ela diversos sentimentos que passam a fazer parte do cotidiano da criança sob tratamento, e dos seus familiares, como por exemplo, medo, tristeza, desânimo, desesperança, pesar, aflição, angústia, inquietação e impaciência. São sentimentos que surgem relacionados ao diagnóstico, à incerteza quanto ao prognóstico e à complexidade do tratamento ${ }^{18}$.

No presente estudo, foram feitos o autorrelato (visão dos pacientes em relação à sua qualidade de vida) e o heterorrelato (visão dos pais em relação à qualidade de vida dos filhos) do questionário PedsQLTM Cancer 3.0 Module. Ao serem comparados, percebe-se que a qualidade de vida relacionada à saúde (QVRS) no heterorrelato encontra-se em declínio, desde a primeira semana do diagnóstico até 30 dias após, diferente do autorrelato que apresenta uma melhora progressiva da qualidade de vida do início e após 30 dias de tratamento. Essa informação corrobora o estudo de $\mathrm{Matziou}^{19}$, no qual é relatado que os pais têm tendência a associar a doença oncológica com o mau prognóstico e a incertezas no futuro, mais do que as crianças.

Neste estudo, nos primeiros sete dias, a criança se encontrava tranquila e os pais, muito ansiosos. No segundo momento (15 dias), ambos se encontravam ansiosos. Já no terceiro (30 dias), pais e filhos estavam menos ansiosos do que no início. Para Matziou ${ }^{19}$, a ansiedade nos tratamentos (como: ir ao médico, ao hospital ou pela espera do médico) está relacionada com um sentimento de ameaça que antecede aos momentos de perigo ou de tensão de causa desconhecida, dependendo da maturidade e do nível cognitivo, ambos os dois muito diferentes entre criança e adultos.

Novamente, por apresentarem impressóes diferentes relacionadas ao tratamento do câncer, pais e crianças demonstraram opinióes opostas no domínio "Comunicaçáo" (questiona se o paciente consegue ou não tirar dúvidas ou responder às perguntas da equipe do hospital). Enquanto os pais percebiam que os pacientes tinham dificuldades para responder ou questionar suas dúvidas, as crianças responderam que era fácil. Tornandose clara a importância de um acompanhamento de uma equipe multidisciplinar nesses casos, com pais e pacientes, com o objetivo de estarem todos juntos em algum momento desmistificando as preocupaçóes.

No estudo de Matziou ${ }^{19}$, foram convidados 149 pacientes jovens com câncer acompanhados pelo 
ambulatório de um hospital da Grécia, sendo divididos em dois grupos: tratamento (pacientes que foram recentemente diagnosticados ou sob tratamento, mas sem recaídas); e sem tratamento (pacientes que ainda estavam realizando consultas após o tratamento do câncer). Utilizaram o Questionário PedsQL $L^{T M} 4.0$ Generic Core Scales para observar a concordância entre as respostas dos pais e dos pacientes. O resultado encontrado foi de que os pacientes jovens apresentam melhor qualidade de vida (74.58) (16.68) em relação aos pais 69.18 (17.91), indo ao encontro do presente estudo. Muitas vezes, os pais acabam projetando nos filhos suas preocupaçóes em relação ao tratamento do câncer, ou então, dependendo da idade, a criança acaba não tendo ideia do que está acontecendo com ela e, por isso (principalmente nos primeiros dias após o diagnóstico), não consegue ver diferença na sua QVRS.

Cabe ressaltar que o número de indivíduos do estudo não foi contemplado em razão do pouco tempo para coletas. Assim como, são poucos estudos voltados para a relação de avaliaçóes prévias (física, comportamental) em crianças e adolescentes que estáo iniciando a quimioterapia, dificultando e limitando, dessa forma, a discussão. Por essa razão, sugere-se que pesquisas similares sejam realizadas, com o objetivo de proporcionar um tratamento adequado para esses pacientes, auxiliando tanto a parte física como a social.

\section{CONCLUSÃO}

A força de preensão palmar nas crianças e adolescentes com câncer diminui progressivamente a partir do início da quimioterapia. Sendo assim, é indispensável o acompanhamento fisioterapêutico na atenção a esses pacientes. Trata-se de um público que apresenta bons resultados para o tratamento, por isso é dever do profissional fisioterapêutico elaborar um programa de exercícios (individual) que proporcione bem-estar e melhore a qualidade de vida desses pacientes desde o momento do diagnóstico.

Em razão do número reduzido da amostra, não houve diferença significativa em relação ao questionário de qualidade de vida. Porém, ao analisar de forma separada os domínios, observaram-se os momentos de maior instabilidade dos filhos e pais, evidenciando a importância de uma equipe multidisciplinar na abordagem desses pacientes e de seus familiares.

\section{CONTRIBUIÇÕES}

Thayze Bairros da Costa contribuiu na concepção e desenho do trabalho; aquisição, análise e interpretação dos dados da pesquisa e redação. Michelle Hagi Frantzeski e Daniela Meirelles do Nascimento contribuíram na concepção e desenho do trabalho; análise interpretação dos dados da pesquisa; redação e revisão crítica com contribuição intelectual; e aprovação final da versão para publicação. Lauro José Gregianin contribuiu na concepçâo e desenho do trabalho e aquisição dos dados de pesquisa.

\section{DECLARAÇÃO DE CONFLITO DE INTERESSES}

Nada a declarar.

\section{FONTES DE FINANCIAMENTO}

Não há.

\section{REFERÊNCIAS}

1. Instituto Nacional de Câncer José Alencar Gomes da Silva. Câncer infantojuvenil. [Internet]. Rio de Janeiro: INCA; [modificaçáo 2018 Nov 21; acesso 2018 Nov 25]. Disponível em: http://www2.inca.gov.br/wps/ $\mathrm{wcm} /$ connect/tiposdecancer/site/home/infantil.

2. Saevarsdottir T, Fridriksdottir N, Gunnarsdottir S. Quality of life and symptoms of anxiety and depression of patients receiving cancer chemotherapy: longitudinal study. Cancer Nurs. 2010;33(1):1-10.

3. Chatterjee K, Zhang J, Tao R, Honbo N, Karliner J. Vincristine attenuates doxorubicin cardiotoxicity. Biochem Biophys Res Commun. 2008;373(4):555-60.

4. Longui, CA. Glucocorticoid therapy: minimizing side effects. J Pediatr. 2007;83(Supl 5):S163-171.

5. Tan SY, Poh BK, Chong HX, Ismail MN, Rahman J, Zarina AL, et al. Physical activity of pediatric patients with acute leukemia undergoing induction or consolidation chemotherapy. Leuk Res. 2013;37(1):14-20.

6. Bianco A, Patti A, Thomas E, Palma R, Maggio MC, Paoli A, Palma A. Evaluation of fitness levels of children with a diagnosis of acute leukemia and lymphoma after completion of chemotherapy and autologous hematopoietics stem cell transplantation. Cancer Med. 2014;3(2):385-389.

7. Dias JA, Ovando AC, Külkamp W, Borges NG Jr. Força de preensão palmar: métodos de avaliação e fatores que influenciam a medida. Rev Bras Cineantropom Desempenho Hum. 2010,12(3):209-216.

8. Silva MGP, Bedor CNG, Alencar KMSA, Curado MP, Moura LTR. Tendências da morbimortalidade por câncer infantojuvenil em um polo de fruticultura irrigada. Cad Saúde Colet. 2018;26(1):38-44.

9. Ward E, DeSantis C, Robbins A, Kohler B, Jemal A. Childhood and adolescent cancer statistics, 2014. CA Cancer J Clin. 2014;64(2):83-103. 
10. Moura PMLS, Moreira D, Caixeta AP. Força de preensão palmar em crianças e adolescentes saudáveis. Rev Paul Pediatr. 2008;26(3):290-4.

11. Götte M, Kesting SV, Winter CC, Rosenbaum D, Boos $\mathrm{J}$. Motor performance in children and adolescents with cancer at the end of acute treatment phase. Eur J Pediatr. 2015;174(6):7 91-9.

12. Chabner BA, Longo DL. Manual de Oncologia de Harrison. 2. ed. Porto Alegre: AMGH Ed; 2015.

13. Rodríguez-Reyes MC, Galván-Canchila DM. Efectos de la quimioterapia en el sistema musculoesquelético de nińos y adolescentes con leucemia linfoblástica aguda. Rev Univ Salud. 2014;16(1):114-121.

14. Díaz-Jaime E, Peñaloza-Ochoa L, Parada-Onoko PM. Cambios electrofisiológicos de la neuropatía periférica por vincristina posteriores a un programa de terapia física en pacientes pediátricos con leucemia linfoblástica aguda. Bol Med Hosp Infant Mex. 2009 nov-dic;66:529-536.

15. Hartman A, Van Den Bos C, Stijnen T, Pieters R. Decrease in peripheral muscle strength and ankle dorsiflexion as long-term side effects of treatment for childhood cancer. Pediatr Blood Cancer. 2008;50(4):833-837.

16. Deisenroth A, Söntgerath R, Schuster AJ, Busch C, Huber G, Eckert K, et al. Muscle strength and quality of life in patients with childhood cancer at early phase of primary treatment. Pediatr Hematol Oncol. 2016 sep;33(6):393-407.

17. Kilgour, RD, Vigano A, Trutschnigg B, Lucar E, Borod M.; Morais JA. Handgrip strength predicts survival and is associated with markers of clinical and functional outcomes in advanced cancer patients. Support Care Cancer. 2013;21(12):3261-3270.

18. Almico T, Faro A. Coping of caregivers of children with cancer in chemotherapy process. Psic Saúde Doenças. 2014;15(3):723-737.

19. Matziou V, Perdikaris P, Feloni D, Moshovi M, Tsoumakas K, Merkouris A. Cancer in childhood: children's and parents' aspects for quality of life. Eur J Oncol Nurs. 2008 jul;12(3):209-216. 